\title{
Successful endoscopic wire-guided balloon dilatation of angulated and tight ileal pouch strictures without fluoroscopy
}

\author{
Emmanuel C. Obusez $\cdot$ Lei Lian • Abigail Oberc $\cdot$ \\ Bo Shen
}

Received: 16 February 2010/Accepted: 18 August 2010/Published online: 11 September 2010

(C) Springer Science+Business Media, LLC 2010

\begin{abstract}
Background Ileal pouch strictures that are visually inaccessible by an endoscope may be balloon-dilated by exchange guide wire across the stricture with the aid of fluoroscopy. We present a technique of wire-guided balloon dilation without fluoroscopy to navigate strictures in the ileal pouch.

Methods A 50-year-old Caucasian female presented with a 24-year history of ulcerative colitis (UC) with restorative proctocolectomy and ileal pouch anal anastomosis (IPAA) for 7 years. She developed Crohn's disease (CD) of the pouch with multiple strictures at the afferent limb of the pouch and a pouch-vaginal fistula. On pouchoscopy, the patient had two strictures at the distal neoterminal ileum, at $10 \mathrm{~cm}$ and $15 \mathrm{~cm}$ proximal to the pouch inlet. In retrospect, the distal stricture was angulated and $1 \mathrm{~cm}$ in length, and the proximal one was ulcerated and pinhole in size, which prevented the passage of an endoscope $(9.8-\mathrm{mm}$ singlechannel, GIF-H180; Olympus Optical, Tokyo, Japan). The stricture number and locations were confirmed by retrograde water-soluble contrast X-ray. There was great difficulty in negotiating the strictures with balloon dilation and
\end{abstract}

hence concern that blind passage of the balloon into the strictures might induce mucosal trauma or perforation. A controlled radial expansion (CRE) wire-guided balloon dilation catheter (CRE TM Single-Use Wire Guided Balloon Dilator; Boston Scientific Microvasive, Natick, MA) was introduced through the scope. Wire exchange technique was applied with help of our endoscopy nurse (A.O.). The guide wire was passed through the strictures without any resistance under endoscopy view. Subsequently, the balloon was introduced across the strictures, and both were successfully dilated to $16 \mathrm{~mm}$ (Videos 1 and 2).

Results The procedure and postprocedure course were uneventful, and patient reported great symptomatic relief. Conclusion Endoscopic guide-wire balloon dilation without fluoroscopic guidance appears to be feasible for CD-related strictures in experienced hands.

Disclosures Authors Emmanuel C Obusez, Abigail Oberc, Lei Lian, and Bo Shen have no conflicts of interest or financial ties to disclose.
Electronic supplementary material The online version of this article (doi:10.1007/s00464-010-1339-9) contains supplementary material, which is available to authorized users.

E. C. Obusez · L. Lian · A. Oberc · B. Shen $(\bowtie)$

Cleveland Clinic Lerner College of Medicine and Pouchitis

Clinic, Digestive Disease Institute, Desk A31, Cleveland Clinic

Foundation, 9500 Euclid Ave, Cleveland, OH 44195, USA

e-mail: shenb@ccf.org 